\title{
Broadband omnidirectional antireflection coating based on subwavelength surface Mie resonators
}

P. Spinelli ${ }^{1}$, M.A. Verschuuren ${ }^{2} \&$ A. Polman ${ }^{1}$

Reflection is a natural phenomenon that occurs when light passes the interface between materials with different refractive index. In many applications, such as solar cells or photodetectors, reflection is an unwanted loss process. Many ways to reduce reflection from a substrate have been investigated so far, including dielectric interference coatings, surface texturing, adiabatic index matching and scattering from plasmonic nanoparticles. Here we present an entirely new concept that suppresses the reflection of light from a silicon surface over a broad spectral range. A two-dimensional periodic array of subwavelength silicon nanocylinders designed to possess strongly substrate-coupled Mie resonances yields almost zero total reflectance over the entire spectral range from the ultraviolet to the near-infrared. This new antireflection concept relies on the strong forward scattering that occurs when a scattering structure is placed in close proximity to a high-index substrate with a high optical density of states.

\footnotetext{
${ }^{1}$ Center for Nanophotonics, FOM Institute AMOLF, Amsterdam, The Netherlands. ${ }^{2}$ Philips Research Laboratories, Eindhoven, The Netherlands. Correspondence and requests for materials should be addressed to A.P. (email: polman@amolf.nl).
} 
M any different ideas have been developed over the years to reduce reflection in optical and optoelectronic devices ${ }^{1,2}$. A single transparent dielectric layer is commonly used as an antireflection (AR) coating. On the basis of destructive interference between the incident and reflected light, perfect optical impedance matching can be achieved for one specific wavelength ${ }^{3}$. Micron-sized pyramidal surface texturing is also widely used in, for example, crystalline silicon solar cells ${ }^{4,5}$. The angular randomization of the scattered light at the surface can increase light absorption in an optical medium up to a factor of $4 n^{2}$, where $n$ is the refractive index of the medium. ${ }^{6,7}$ Surface structures composed of periodic or random arrays of semiconductor nanowires and nanopyramids have also proven to reduce reflection over a broad range of wavelengths and angles of incidence ${ }^{7-15}$. The reduced reflection is caused by multiple scattering of light as well as adiabatic coupling due to a gradually increasing refractive index towards the substrate surface ${ }^{16-21}$. Recently, we have shown that optimized arrays of metal nanoparticles reduce reflection of silicon in the range of wavelengths relevant for solar applications ${ }^{22,23}$. Preferential forward scattering by the plasmonic point scatterers ${ }^{24}$ strongly reduces the reflection, thus allowing almost perfect impedance matching of sunlight into a Si wafer.

Here we present an entirely new concept to eliminate reflection from a silicon wafer that makes use of much simpler nanostructures than the ones reported before, does not involve metals, and can be applied to any high-index material. It is based on the fact that dielectric nanoscatterers possess geometrical Mie resonances with scattering cross-sections larger than the geometrical ones ${ }^{25,26}$. A recent paper by Cao et al..$^{27}$ has shown that Mie resonances provide a very efficient mechanism for light absorption in Si nanowire-based solar cells. In that work, however, Si nanowires were on a glass substrate and only absorption in the nanowires was considered. In this paper, we demonstrate that light that is stored in Mie resonances can also be very efficiently coupled to a Si substrate. From a fundamental point of view this light coupling mechanism, based on weak (lossy) Mie resonances, is entirely different than all other methods commonly known in the field of AR coatings. We demonstrate experimentally that an array of low aspect-ratio Si nanocylinders etched into a Si wafer shows an average reflectivity as low as $1.3 \%$ in the $450-900 \mathrm{~nm}$ spectral range. The strongly reduced reflectivity is observed for angles of incidence up to $60^{\circ}$. The monolithic nature of the nanostructures makes this novel AR concept easily applicable to a wide range of devices, including (ultra-thin) solar cells based on any high-index semiconductor ${ }^{28}$.

\section{Results}

Mie scattering on a Si substrate. Figure 1a shows the calculated scattering cross-section spectra, normalized to the geometrical cross-section, for a Si sphere in air (blue) and a Si sphere (green) and cylinder (red) on top of a Si substrate. The scattering spectrum for the sphere in air shows two distinct resonances corresponding to the first- and second-order Mie modes. These geometrical resonances reflect the fact that the particle acts as a nano-cavity for light. The scattering cross-section at resonance is more than eight times larger than the geometrical cross-section, and the cavity quality factor $(Q=10)$ is determined by radiation losses from the cavity mode. The two scattering resonances are clearly visible when a Si substrate is introduced below the spherical particle (green line), indicating only weak coupling of the well-confined Mie modes to the substrate. However, when a cylindrical nanoparticle is placed on the $\mathrm{Si}$ substrate, the resonances broaden and merge into a broadband feature (red line). This behaviour is due to the introduction of a leaky channel into the substrate for the light that is resonantly confined in the particle. To understand this mechanism, Figure $1 \mathrm{~b}-\mathrm{g}$ show the calculated distribution of light (electric field intensity, colour scale) inside a Si sphere in air (Fig. 1b,e), a Si sphere on a Si substrate a

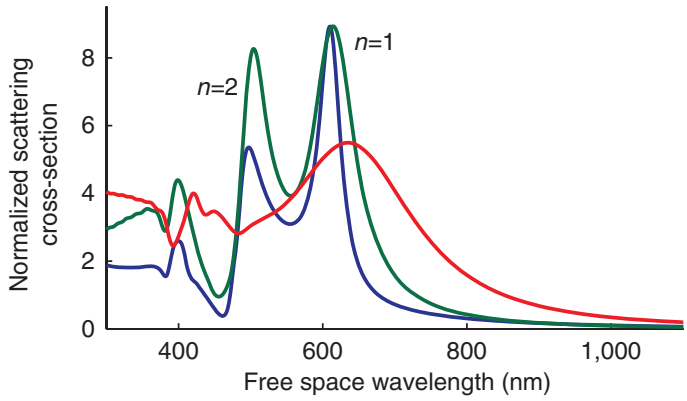

b
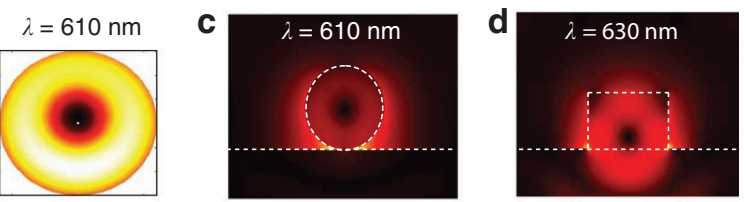

e
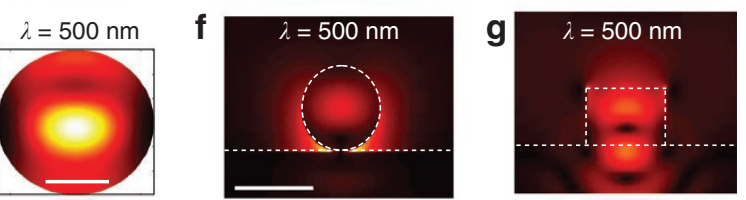

Figure 1 | Mie scattering on a Si wafer. (a) Scattering cross-sections, normalized to the geometrical cross-section, for a Si sphere in air (blue), a Si sphere on a Si substrate (green) and a Si cylinder on a Si substrate (red). The spheres have diameter $d=150 \mathrm{~nm}$, the cylinder has an in-plane diameter of $150 \mathrm{~nm}$ and height of $100 \mathrm{~nm}$. Mie resonances of first $(n=1)$ and second $(n=2)$ order are indicated in the figure. $(\mathbf{b}-\mathbf{g})$ Electric field intensity (colour scale) in a cross-section of the particle for a sphere in air $(\mathbf{b}, \mathbf{e})$, a sphere on substrate $(\mathbf{c}, \mathbf{f})$ and a cylinder on substrate $(\mathbf{d}, \mathbf{g})$, for Mie modes of first (b-d) and second (e-g) order. The wavelengths considered for these calculations are indicated in each panel. The field distribution for the cylinder at resonance overlaps with the substrate, thus introducing a loss channel for the light confined in the particle that broadens the resonances. Plots for a sphere in air are calculated with Mie theory; plots for particles on a substrate are simulated. The scale bar in e represents $50 \mathrm{~nm}$, and refers to $\mathbf{b}$ and $\mathbf{e}$. The scale bar in $\mathbf{f}$ represents $150 \mathrm{~nm}$ and refers to $\mathbf{c}, \mathbf{d}, \mathbf{f}$ and $\mathbf{g}$.

(Fig. 1c,f) and a Si cylinder on a Si substrate (Fig. 1d,g) for Mie modes of first (Fig. 1b-d) and second (Fig. 1e-g) order. Comparison of the field distributions shows that the geometrical modes inside a spherical Si particle are nearly unaffected by the presence of the substrate and light in both cases is well confined inside the particle. However, for a cylindrical particle the field distribution of the Mie resonances has a clear overlap with the $\mathrm{Si}$ substrate. Both direct near-field absorption in the substrate and radiative emission into the substrate introduce a loss channel for the light confined in the particle, thus yielding broadening of the resonances in the scattering cross-section spectrum (Q 5; Supplementary Fig. S1).

Ultra-low reflectivity. The field distributions in Figure 1b-g suggest that a cylindrical Si nanoparticle can effectively couple light into a Si substrate. To study this further we perform simulations of the reflectance spectra of cylindrical nanopillar (NP) arrays with different NP dimensions. Figure $2 \mathrm{a}$ shows the simulated total reflectance spectra from a square array of Si NPs spaced by $500 \mathrm{~nm}$, for cylinder diameters of 75, 100 and $125 \mathrm{~nm}$. The reflectance from a bare flat Si surface (35-60\%) is also shown for comparison (black line). The data in Figure $2 \mathrm{a}$ show that the dielectric particle array reduces the reflectance of a Si surface over the entire spectral range shown. The key role of the Mie resonances in the reduced reflection is clear from the fact that broad reflection dips are observed 

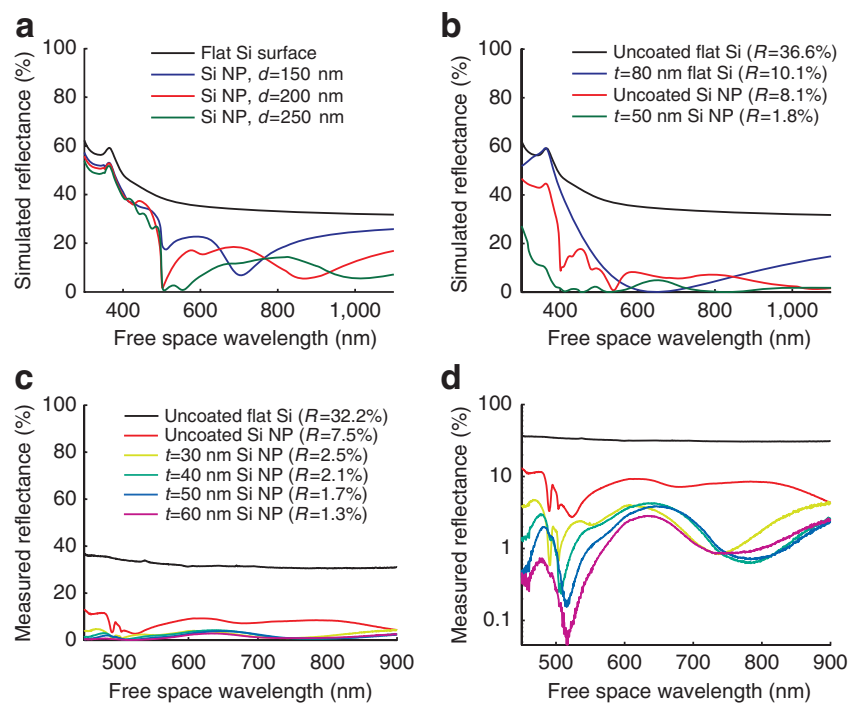

Figure 2 | Ultra-low reflectivities. (a) Simulated reflection spectra from a regular square array of Si NPs spaced by $500 \mathrm{~nm}$, for cylinder diameters of 150 (blue), 200 (red) and $250 \mathrm{~nm}$ (green) with a height of $150 \mathrm{~nm}$. Reflectance from a flat $\mathrm{Si}$ surface is also shown for comparison (black). The Si NP arrays reduce the reflectivity over the entire spectrum. The broad dip in reflectivity that redshifts for increasing particle diameter is due to enhanced forward scattering from the Mie resonances in the particle. (b) Simulated reflection spectra for a bare flat Si substrate (black), a flat $\mathrm{Si}$ substrate coated with a standard $\mathrm{Si}_{3} \mathrm{~N}_{4}$ antireflection coating (blue, thickness $t=80 \mathrm{~nm}$ ), a Si surface with bare Si nanostructures on top (red) and a Si surface with Si nanostructures on top coated with an optimized $\mathrm{Si}_{3} \mathrm{~N}_{4}$ layer (green, $t=50 \mathrm{~nm}$ ). For each configuration the average reflectivity, $R$, weighted with the AM1.5 solar spectrum in the $300-1,100 \mathrm{~nm}$ spectral range, is indicated. (c) Measured total reflectivity of a bare Si wafer (black), an uncoated Si NP array (red) and four Si NP array coated with $\mathrm{Si}_{3} \mathrm{~N}_{4}$ layers of different thicknesses, $t$ (colours). The Si NPs have a diameter of $125 \mathrm{~nm}$, height of $150 \mathrm{~nm}$ and are spaced by $450 \mathrm{~nm}$. For each configuration the average reflectivity, $R$, weighted with the AM1.5 solar spectrum in the $450-900 \mathrm{~nm}$ spectral range, is indicated. Reflectance is reduced over the entire spectral range, due to coupling of the Mie resonant scattering to the Si substrate. (d) The same reflectivity data plotted in logarithmic scale. Individual curves are described in legend to Figure $3 c$. The effect of the Mie resonance is visible in the broad dip in reflectivity observed in the $700-800 \mathrm{~nm}$ range.

on resonance, at wavelengths $(700,830$ and $1,000 \mathrm{~nm})$ that increase with NP diameter $(150,200$ and $250 \mathrm{~nm})$. This redshift for increasing diameter is similar to that observed for Mie resonances for isolated particles with increasing diameter (Supplementary Fig. S1). The sharp decrease in reflectivity at the wavelength of $500 \mathrm{~nm}$, that is, equal to the array pitch, is explained by a Rayleigh anomaly ${ }^{29}$ from the grating formed by the regular array of nanoparticles (Supplementary Fig. S2).

The strong coupling of light on resonance is explained by the strong forward scattering of light from the resonant Mie scatterers, due to the high optical mode density of the high-index Si substrate ${ }^{30}$. The resonant nature of this effect leads to a large cross-section, so that even an array of scatterers covering only $30 \%$ of the surface area leads to nearly complete interaction with the incoming plane wave. Figure $2 \mathrm{~b}$ shows the simulated reflectivity spectra for a bare flat $\mathrm{Si}$ substrate (black), a flat $\mathrm{Si}$ substrate coated with a standard $80 \mathrm{~nm}$ $\mathrm{Si}_{3} \mathrm{~N}_{4}$ antireflection coating (blue), a Si surface with bare Si NP on top (red) and a Si surface with Si NP on top coated with a $50 \mathrm{~nm}$ $\mathrm{Si}_{3} \mathrm{~N}_{4}$ layer (green). Given its index $(n=2.0), \mathrm{Si}_{3} \mathrm{~N}_{4}$ is the optimum AR coating for a $\mathrm{Si}$ wafer $(n=3.5-4.0$ in the visible-to-near-infrared spectral range). As can be seen in the figure, it only leads to vanishing reflectivity for a specific wavelength $(650 \mathrm{~nm}$ in Fig. 2b), determined by the $\mathrm{Si}_{3} \mathrm{~N}_{4}$ layer thickness. The bare array of Si NPs shows a broadband reduced reflectivity spectrum that is lower than that for the standard AR coating above $850 \mathrm{~nm}$ and below $550 \mathrm{~nm}$, and higher in the range $550-850 \mathrm{~nm}$. Both spectra show a large increase in reflectivity for wavelengths below $500 \mathrm{~nm}$. A $\mathrm{Si}_{3} \mathrm{~N}_{4}$ layer with properly chosen thickness can be used as a coating, conformal to the nanostructures, to compensate for the losses in this spectral region. The reflectance spectrum from a coated array of NPs (Fig. 2b, green) shows that reflectivity can be reduced down to $<2 \%$ over the entire spectral range from 400 to $1,100 \mathrm{~nm}$, with the exception of a small peak around $650 \mathrm{~nm}$. The low reflectivity stems from the combined effect of the strongly coupled Mie resonances in the red and nearinfrared part of the spectrum and the interference antireflection effect from the $\mathrm{Si}_{3} \mathrm{~N}_{4}$ layer, tuned for the blue part of the spectrum. Figure $2 \mathrm{~b}$ also shows the average reflectivities for each configuration, obtained by weighting over the AM1.5 solar spectrum in the $300-1,100 \mathrm{~nm}$ spectral range. As can be seen, both coated (1.8\%) and uncoated NP arrays (8.1\%) show lower reflectivity than the standard $80 \mathrm{~nm} \mathrm{Si}_{3} \mathrm{~N}_{4}$ coating (10.3\%).

Black silicon. The AR properties of Si NP arrays have been studied experimentally using $\mathrm{Si}(100)$ wafers, on which square arrays of Si nanocylinders (250 nm diameter, $150 \mathrm{~nm}$ height, $450 \mathrm{~nm}$ pitch) were fabricated using substrate conformal soft-imprint lithography in combination with reactive ion etching (RIE). The total hemispherical reflectivity was measured using an integrating sphere, with an angle of incidence (AOI) of $8^{\circ}$ off the surface normal. Figure $2 \mathrm{c}$ shows the measured total reflectivity spectrum of a bare Si wafer (black), of a bare Si NP array (red) and four Si NP arrays coated with different $\mathrm{Si}_{3} \mathrm{~N}_{4}$ layer thicknesses (green to purple colour lines). All geometries show a reduced reflectivity with respect to the bare Si wafer, over the entire spectral range. The array coated with a 60$\mathrm{nm}$ thick $\mathrm{Si}_{3} \mathrm{~N}_{4}$ layer (purple line) shows a reflectivity $<3 \%$ over the entire spectral range $450-900 \mathrm{~nm}$. The AM1.5-averaged reflectance from a bare Si substrate (32.2\%, black) is reduced to $7.5 \%$ by pattering the Si surface with an optimized Si NP array (red). Combining $\mathrm{Si} \mathrm{NPs}$ and $\mathrm{Si}_{3} \mathrm{~N}_{4}$ coating leads to an average reflectivity as low as $1.3 \%$ (purple). This value is in excellent agreement with the calculated data in Figure 2b, taking into account the different spectral range over which the average reflectivity is calculated (Supplementary Figs S3 and S4). Figure 2d shows the same reflectivity spectra plotted on a logarithmic scale. The broad dip in the reflectivity spectra at wavelengths between 650 and $850 \mathrm{~nm}$ is attributed to the first-order coupled Mie resonances. The sharp dips at a wavelength of about $510 \mathrm{~nm}$ are consistent with a Rayleigh anomaly for light incident with an AOI of $8^{\circ}$ (Supplementary Fig. S5).

With the reflection of light effectively vanished, light is then either transmitted into the Si substrate or absorbed in the NPs. For example, for silicon in the $800-1,000 \mathrm{~nm}$ spectral range the linear absorption over the thickness of the nanocylinders is $<1 \%$; it is enhanced in the cavity by the quality factor Q 3. Indeed, simulations (Supplementary Fig. S6) show that in this spectral range absorption in the NPs is negligible and the NPs behave as almost pure scatterers (that is, the albedo is almost 1). For the ultraviolet/blue spectral range, $\mathrm{Si}$ is strongly absorbing and light is absorbed inside the Si nanocylinders. Indeed, an optimized solar cell design based on substrate-coupled Mie resonators will have the $\mathrm{p}-\mathrm{n}$ junction integrated inside the nanoparticles, which can easily be done using gas-phase doping.

Figure 3a shows a photograph of a bare flat 4-inch Si wafer compared to a 4-inch Si wafer that has been patterned over the entire wafer area with an optimized Si NP array and overcoated with a 60nm-thick $\mathrm{Si}_{3} \mathrm{~N}_{4}$ layer. The photograph shows a clear visual effect of the reduced reflectivity due to the patterning of the Si wafer surface with resonant Mie scatterers. Figure 3 shows a scanning electron 
microscopy image of a bare (Fig. $3 \mathrm{~b}$ ) and a $60-\mathrm{nm} \mathrm{Si}_{3} \mathrm{~N}_{4}$-coated (Fig. 3c) NP array. The sample shown in Figure 3 was fabricated using interference lithography and soft-imprint lithography, demonstrating that this new AR coating design can be applied at largearea using standard inexpensive processes.

Angle-resolved reflectivity. To be relevant for, for example, photovoltaic applications, an AR coating must show low reflectivity over a wide range of angles of incidence and for both polarizations of light. Figure 4 shows the specular reflectivity measured as a function of AOI, for wavelengths of $514 \mathrm{~nm}$ (Fig. 4a,d), $632 \mathrm{~nm}$ (Fig. 4b,e) and $405 \mathrm{~nm}$ (Fig. 4c,f). Figure $4 \mathrm{a}-\mathrm{c}$ shows reflectance for s- (solid
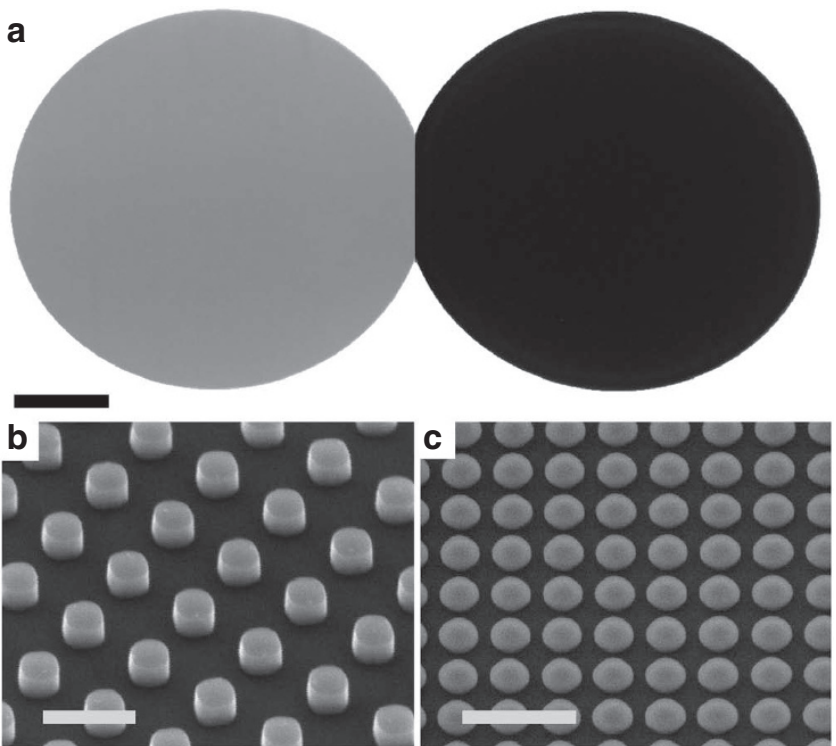

Figure 3 | Black silicon. (a) Photograph of a bare flat 4-inch Si wafer (left) and a 4-inch Si wafer fully imprinted with an optimized (250 nm diameter, $150 \mathrm{~nm}$ height, $450 \mathrm{~nm}$ pitch) Si NP array and overcoated with a 60-nmthick $\mathrm{Si}_{3} \mathrm{~N}_{4}$ layer (right). Scale bar represents 1 inch. (b) Scanning electron microscopy image taken under an angle of $40^{\circ}$ of a bare Si NP array (scale bar represents $500 \mathrm{~nm}$ ) and (c) a Si NP array coated with a 60-nm-thick $\mathrm{Si}_{3} \mathrm{~N}_{4}$ layer (scale bar represents $1 \mu \mathrm{m}$ ). symbols) and p-polarized (open symbols) incident beams, whereas Figure $4 \mathrm{~d}-\mathrm{f}$ shows the average reflectivity for s- and p-polarizations, plotted on a logarithmic scale. In each graph, reflectivities from a bare $\mathrm{Si}$ wafer (black lines), a flat $\mathrm{Si}$ wafer with a $60-\mathrm{nm}$ standard $\mathrm{Si}_{3} \mathrm{~N}_{4}$ coating (red) and a 60 -nm-thick $\mathrm{Si}_{3} \mathrm{~N}_{4}$-coated $\mathrm{NP}$ array (blue) are shown. At a wavelength of $514 \mathrm{~nm}$, the $60 \mathrm{~nm}$ standard coating and the coated NP array show a total reflectivity for near-normal incidence of 0.1 and $0.05 \%$, respectively (Supplementary Fig. S3a). Figure $4 \mathrm{a}$ shows that for both samples and polarizations the reflectivity remains below $1 \%$ in a wide range of AOI. While no difference between the standard coating and the Si NP array is seen for the s-polarization, the Si NP array shows lower reflection of p-polarized light at large angles. Averaging the two polarizations (Fig. 4d) yields similar results for the standard and the Si NP coatings, with better AR properties from the latter for angles above $14^{\circ}$. At wavelengths of $632 \mathrm{~nm}$ (Fig. 4b) and $405 \mathrm{~nm}$ (Fig. 4c), the Si NP array shows lower reflectivity than the standard $\mathrm{Si}_{3} \mathrm{~N}_{4}$ coating, for both polarizations and over the entire range of AOI. As Figure $4 \mathrm{e}$ and $\mathrm{f}$ shows, the low reflectivity from the coated $\mathrm{Si} \mathrm{NP}$ array is maintained over the entire angular range up to $60^{\circ}$.

\section{Discussion}

Subwavelength resonant Mie scatterers at the surface of a Si wafer cause the reflection of light from the wafer to vanish. The resonant Mie modes have large scattering cross-sections, leading to strong interaction with the incident light. Coupling of these modes to the substrate leads to strong preferential forward scattering due to the high-mode density in the high-index substrate. Full-wafer Si nanocylinder arrays were fabricated using a soft-imprint technique capable of large scale, high-fidelity surface patterning. Total reflectance spectroscopy shows an average reflectivity of only $1.3 \%$ over the $450-900 \mathrm{~nm}$ spectral range. The strongly reduced reflectivity is observed for a broad range of angles of incidence up to $\pm 60^{\circ}$. Owing to the monolithic nature of the Mie scatterers, this novel antireflection concept is easily applicable to any high-index material. It can readily find application in solar cells, including thin-film designs on which the standard texturing cannot be applied.

\section{Methods}

Numerical calculations. The scattering cross-section spectra and field distributions shown in Figure 1 have been calculated using Mie theory ${ }^{26}$ (blue line in Fig. la and Fig. $1 \mathrm{~b}$ and e) and finite-difference time-domain (FDTD) calculations (green and red lines in Fig. 1a, and Fig. 1c,d,f and g). For a definition of scatter- a

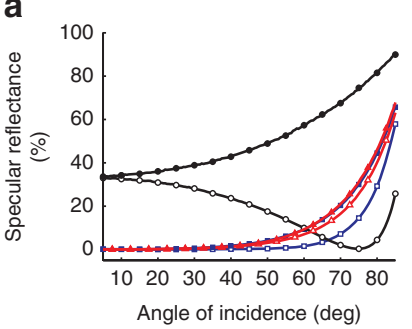

d

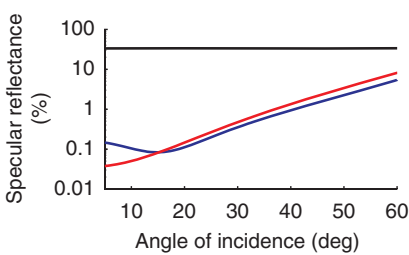

b

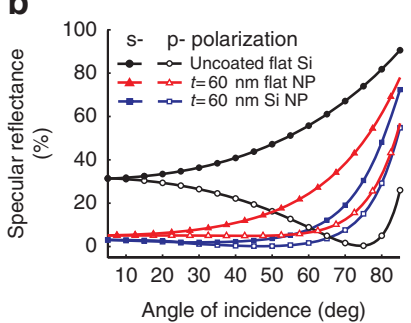

e

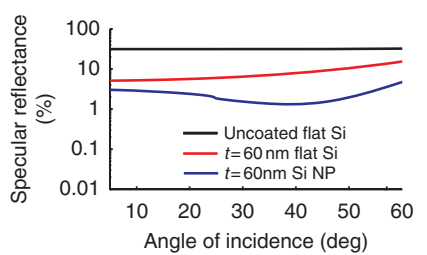

C

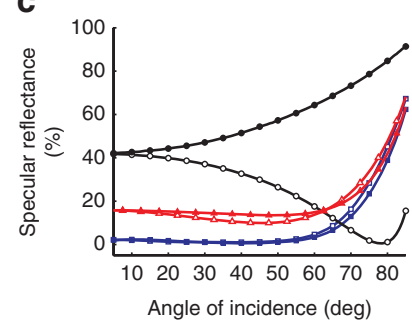

f

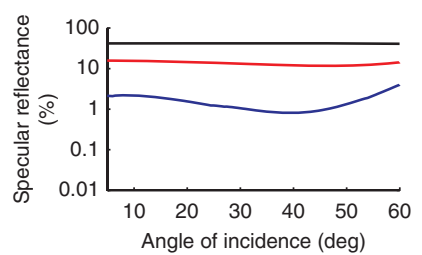

Figure 4 | Angle resolved reflectivity. Specular reflectivity measured as a function of AOI, for wavelengths of $514 \mathrm{~nm}(\mathbf{a}, \mathbf{d}), 632 \mathrm{~nm}(\mathbf{b}, \mathbf{e})$ and $405 \mathrm{~nm}$ $(\mathbf{c}, \mathbf{f})$. Panels in the top row show results for s- (solid symbols) and p-polarized (open symbols) incident beam, whereas the bottom row show an average of $\mathrm{s}-$ and p-polarizations, plotted on a logarithmic scale. In each graph, reflectivities from a bare Si wafer (black lines), a 60-nm standard $\mathrm{Si}_{3} \mathrm{~N}_{4}$ coating (red) and a coated NP array (blue) are shown. The excellent AR properties of Si NP arrays are maintained over the entire range of $A O I$ from $-60^{\circ}$ to $+60^{\circ}$. 
ing cross-section please refer to ref. 26, chapter 2 . A commercial software package was used to perform FDTD calculations ${ }^{31}$. A simulation box size of $1 \times 1 \times 0.8 \mu \mathrm{m}$ with perfectly matched layer conditions on every boundary was used. The light source was a broadband $(300-1100 \mathrm{~nm})$ plane wave, with normal incidence to the substrate. The mesh grid was set to $5 \mathrm{~nm}$ over the entire simulation volume, with a refinement $(1 \mathrm{~nm})$ over the volume occupied by the particle. A total-field scattered-field method was used to directly calculate the scattered power by means of frequency-domain transmission monitors positioned in the scattered field region. A two-dimensional frequency-domain field monitor cross-cutting the particle in its centre was used to calculate the electric field intensity distribution (as shown in Fig. $1 \mathrm{c}, \mathrm{d}, \mathrm{f}$ and g). FDTD simulations were also used to study the AR properties of Si NP arrays on a Si substrate (Fig. 2a and b). In this case, the box size was equal to $p \times p \times 0.6 \mu \mathrm{m}$, where $p$ is the array pitch, and periodic boundary conditions were used in the in-plane directions. The reflectivity spectra were directly calculated with a frequency-domain transmission monitor placed above the plane-wave source. Optical constants of $\mathrm{Si}$ and $\mathrm{Si}_{3} \mathrm{~N}_{4}$ were taken from the literature ${ }^{32}$.

Fabrication of Si NP arrays. Substrate conformal imprint lithography was used to fabricate large area Si NP arrays. This soft-imprint fabrication technique is capable of large-scale, high-fidelity surface patterning and is fully compatible with standard techniques used in, for example, solar cell manufacturing. It uses a rubber (PDMS) stamp in which a pattern is moulded from a master Si wafer that is patterned using optical interference lithography or electron beam lithography. The rubber stamp is used to replicate the patterns in a sol-gel layer spin-coated on the Si wafer. The patterned sol-gel layer is then used as a mask for a RIE process that results in the formation of Si nanocylinders in the Si wafer. For this work, master template containing a $4 \times 4 \mathrm{~mm}^{2}$ array of $250 \mathrm{~nm}$ wide, $150 \mathrm{~nm}$ high Si NPs with array pitch of $450 \mathrm{~nm}$ was fabricated on a 4 -inch $\mathrm{Si}(100)$ wafer using electron beam lithography and etching. The pattern in the sol-gel was transferred to the Si wafer with two-step RIE: $\mathrm{N}_{2}$ and $\mathrm{CF}_{4}$ gas mixture was used to etch through the sol-gel layer, and $\mathrm{N}_{2}$ and $\mathrm{Cl}_{2}$ gases were used to etch the $\mathrm{Si}$ wafer. The sol-gel etch mask was removed with diluted $\mathrm{HF}$ after which the samples were coated with $\mathrm{Si}_{3} \mathrm{~N}_{4}$ layers of different thicknesses using low-pressure chemical vapour deposition at $850^{\circ} \mathrm{C}$. The 4 -inch wafer shown in the photograph of Figure 3 was fabricated with a similar process, where the master pillar pattern was made using optical interference lithography with a 266-nm wavelength laser.

Optical measurements. The total reflectivity spectra in Figure $2 c, d$ were measured in a Labsphere 4-inch integrating sphere, using a supercontinuum broadband light source (Fianium SC450-A, spectral range $0.45-2 \mu \mathrm{m}$ ). The beam spot on the sample was $1 \mathrm{~mm}$ in diameter. Light from the integrating spheres was collected with a multimode optical fibre and sent to a spectrometer, comprising a spectrograph (Acton, SpectraPro 300i) and a Si CCD array (Princeton Instruments). The data were confirmed by measurements in a PerkinElmer Lambda950 spectrophotometer, using a tungsten-halogen lamp and deuterium lamp as sources, a PM R6872 detector for the ultraviolet-visible spectral range and a Peltier-cooled $\mathrm{PbS}$ detector for near-infrared range.

Specular reflectivity for varying AOI (Fig. 4 and Supplementary Fig. S5) was measured in a double-rotation stage (Huber), where the sample was mounted in the inner stage and a power metre (Thorlabs, PM300) on the outer one. For the measurements at the $632 \mathrm{~nm}$ wavelength, a Helium-Neon laser source and a Thorlabs LPVIS 100 broadband linear polarizer were used (incident power on the sample: $4 \mathrm{~mW}$ ); an argon-krypton laser source and a ColorPol VIS500 linear polarizer $(10 \mathrm{~mW}$ on the sample) were used for the $514 \mathrm{~nm}$ wavelength; a diode laser source and a Thorlabs LPUV 050 polarizer ( $3 \mathrm{~mW}$ on the sample) for the $405 \mathrm{~nm}$ wavelength. In all cases, the beam spot size on the sample was $1 \mathrm{~mm}$ in diameter

\section{References}

1. Nature's guiding light. Nat. Photon 2, 639 (2008).

2. Gevaux, D. Reflection! What reflection? Nat. Photon 1, 186 (2007).

3. Macleod, H. A. Thin-Film Optical Filters (Elsevier, 1969).

4. Lamers, M.W.P.E. et al. $17.9 \%$ Metal-wrap-through mc-Si cells resulting in module efciency of $17.0 \%$. Prog. Photovolt: Res. Appl. 20, 62-73 (2012).

5. Southwell, W. H. Pyramid-array surface-relief structures producing antireflection index matching on optical surfaces. J. Opt. Soc. Am. 8, 549-553 (1991).

6. Yablonovitch, E. Intensity enhancement in textured optical sheets for solar cells. IEEE Trans. El. Dev. 29, 300-305 (1982).

7. Yablonovitch, E. Statistical ray optics. J. Opt. Soc. Am. 72, 899-907 (1982)

8. Clapham, P. B. \& Hutley, M. C. Reduction of lens reflection by the "Moth Eye" principle. Nature 244, 281-282 (1973).

9. Parker, A. R. \& Townley, H. E. Biomimetics of photonic nanostructures. Nat. Nanotech. 2, 347-353 (2007).

10. Huang, Y. et al. Improved broadband and quasi omnidirectional anti-reflection properties with biomimetic silicon nanostructures. Nat. Nanotech. 2, 770-774 (2008).
11. Xi, J.- Q. et al. Optical thin-film materials with low refractive index for broadband elimination of Fresnel reflection. Nat. Photon 1, 176-179 (2007).

12. Her, T.- H., Finlay, R. J., Wu, C., Deliwala, S. \& Mazur, E. Microstructuring of silicon with femtosecond laser pulses. Appl. Phys. Lett. 73, 1673-1675 (1998).

13. Branz, H. M. et al. Nanostructured black silicon and the optical reflectance of graded-density surfaces. Appl. Phys. Lett. 94, 231121 (2009).

14. Yuan, H.- C. et al. Efficient black silicon solar cell with a density-graded nanoporous surface: optical properties, performance, limitations, and design rules. Appl. Phys. Lett. 95, 123501 (2009).

15. Koynov, S., Brandt, M. S. \& Stutzmann, M. Black nonreecting silicon surfaces for solar cells. Appl. Phys. Lett. 88, 203107 (2006).

16. Zhu, J. et al. Optical absorption enhancement in amorphous silicon nanowire and nanocone arrays. Nano Lett. 9, 279-282 (2009).

17. Zhu, J., Hsu, C.- M., Yu, Z., Fan, S. \& Cui, Y. Nanodome solar cells with efficient light management and self-cleaning. Nano Lett. 10, 1979-1984 (2010).

18. Hu, L. \& Chen, G. Analysis of optical absorption in silicon nanowire arrays for photovoltaic applications. Nano Lett. 7, 3249-3252 (2007).

19. Diedenhofen, S. L. et al. Broadband and omnidirectional antireflection coating based on semiconductor nanorods. Adv. Mater. 21, 973-978 (2009).

20. Muskens, O. L., Gómez Rivas, J., Algra, R. E., Bakkers, E.P.A.M. \& Lagendijk, A. Designing light scattering in nanowire materials for photovoltaic applications. Nano Lett. 8, 2638-2642 (2008).

21. Diedenhofen, S. L., Janssen, O. T. A., Grzela, G., Bakkers, E.P.A.M. \& Gómez Rivas, J. Strong geometrical dependence of the absorption of light in arrays of semiconductor nanowires. ACS Nano 5, 2316-2323 (2011)

22. Spinelli, P. et al. Optical impedance matching using coupled metal nanoparticle arrays. Nano Lett. 11, 1760-1765 (2011).

23. Atwater, H. A. \& Polman, A. Plasmonics for improved photovoltaic devices. Nat. Mater. 9, 205-213 (2010).

24. Catchpole, K. R. \& Polman, A. Design principles for particle plasmon enhanced solar cells. Appl. Phys. Lett. 93, 191113 (2008)

25. Mie, G. Beitrge zur Optik trber Medien, speziell kolloidaler Metallsungen. Ann. Phys. 330, 377-445 (1908).

26. Bohren, C. F. \& Huffman, D. R. Absorption and Scattering of Light by Small Particles (Wiley, 2008).

27. Cao, L. et al. Semiconductor nanowire optical antenna solar absorbers. Nano Lett. 10, 439-445 (2010).

28. Pillai, S., Catchpole, K. R., Trupke, T. \& Green, M. A. Surface plasmon enhanced silicon solar cells. J. Appl. Phys. 101, 093105 (2007).

29. Rayleigh, J. W. S. Note on the remarkable case of diffraction spectra discovered by Prof. Wood. Philos. Mag. 14, 60-65 (1907).

30. Kippenberg, T. J., Tchebotareva, A. L., Kalkman, J., Polman, A. \& Vahala, K. J. Purcell-factor-enhanced scattering from Si nanocrystals in an optical microcavity. Phys. Rev. Lett. 103, 027406 (2009).

31. FDTD Solutions 7.5 (Lumerical, 2011).

32. Palik, E. D. Handbook of Optical Constants of Solids (Academic, 1985).

\section{Acknowledgements}

We are grateful to Femius Koenderink for the Matlab code used for the Mie theory calculations, Robert van de Laar for sample fabrication at Mi-Plaza/Philips and Grzegorz Grzela for the help with optical measurements. This work is part of the research programme of the Foundation for Fundamental Research on Matter (FOM), which is financially supported by the Netherlands Organization for Fundamental Research (NWO). It is also funded by the European Research Council. This work is also part of the Global Climate and Energy Project.

\section{Author contributions}

P.S. did the theoretical modelling and the reflectivity measurements. M.A.V. coordinated the fabrication of samples at Philips Research. A.P. supervised the research. All authors contributed to the editing of the manuscript.

\section{Additional information}

Supplementary Information accompanies this paper at http://www.nature.com/ naturecommunications

Competing financial interests: The authors declare no competing financial interests

Reprints and permission information is available online at http://npg.nature.com/ reprintsandpermissions/

How to cite this article: Spinelli, P. et al. Broadband omnidirectional antireflection coating based on subwavelength surface Mie resonators. Nat. Commun. x:x doi: $10.1038 /$ ncomms1691 (2012).

License: This work is licensed under a Creative Commons Attribution-NonCommercialNoDerivative Works 3.0 Unported License. To view a copy of this license, visit http:// creativecommons.org/licenses/by-nc-nd/3.0/ 